\title{
Cylindrically symmetric cosmologies in Lyra geometry
}

\author{
J.K. Singh
}

Received: 13 May 2008 / Accepted: 25 June 2008 / Published online: 22 July 2008

(C) The Author(s) 2008. This article is published with open access at Springerlink.com

\begin{abstract}
A class of new exact solutions of the Einstein field equations have been investigated for stationary cylindrically symmetric space-time around a local cosmic string in the theory based on Lyra's geometry in normal gauge in the presence and absence of an electromagnetic field. The cosmological solutions have been analyzed through various physical and geometrical parameters. It has also been shown that the solutions are space-time inhomogeneous and filled with charged dust.
\end{abstract}

Keywords Local cosmic string · Lyra geometry ·

Electromagnetic field

PACS $04.20 . \mathrm{Jb} \cdot 04.50 .+\mathrm{h} \cdot 98.80 . \mathrm{Cq}$

\section{Introduction}

The cosmological models, which are spatially homogeneous and inhomogeneous, have a significant role in the description of the universe in the early stages of its evolution. It has been a subject of considerable interest of cosmologists to study alternative theories of gravitation. The most important among them proposed by Lyra (1951). Lyra introduced a gauge function into the structure-less manifold as a result of which a displacement field arises naturally. Several authors have studied cosmology in Lyra's geometry with a constant as well as a time-independent displacement field. Halford (1970) studied Robertson-Walker models in Lyra's

J.K. Singh $(\bowtie)$

School of Applied Sciences, Netaji Subhas Institute

of Technology, New Delhi 110 078, India

e-mail: jainendrrakumar@rediffmail.com geometry for a time-independent gauge function. Beesam (1988) investigated FRW models in the Lyra manifold with a time-dependent displacement field. Singh and Singh (1991) studied FRW models in the Lyra's manifold with a constant deceleration parameter, which are relevant to the study of inflationary cosmology. Singh and Shri Ram (1997) discussed the spatially homogeneous Bianchi type-I metric in a different basic form and obtained exact solutions of Einstein field equations in vacuum and in the presence of stiff-matter in the normal gauge when the displacement field is timedependent.

It has also been a subject of interest of many cosmologists to investigate the general problems of rotating cosmological models as well as completely static distribution of charged dust in general relativity (De and Raychaudhury 1968). Som and Raychaudhury (1968) obtained a family of stationary cylindrically symmetric solutions of the EinsteinMaxwell equations corresponding to a charged dust distribution in rigid rotation. Singh (2003) presented an exact cylindrically symmetric solution to Einstein equations when the sources are two perfect fluids. It is also shown that all co moving two-fluid solutions are highly space-time inhomogeneous and filled with charged dust in the presence of an electromagnetic field.

The existence of a large-scale network of strings in the early universe is not a contradiction with the present day observations of the universe, and it can be used to introduce density fluctuations that might explain the problem of galaxy formation (Kibble 1976). The presence of the strings in the early universe can be explained using grand unified theories (GUTs) (Kibble 1976). Kibble (1976) first proposed the idea of a cosmic string. A cosmic string is an infinitely long line with high mass formed as a consequence of macroscopic topological defects. Zeldovich (1980) and Vilenkin 
(1981) indicated that a cosmic string can play an important role in explaining the formation of galaxies. Vilenkin (1981) calculated the metric around a local string in the linear approximation of general relativity, and obtained a space-time metric, which is flat but with a deficit angle $\Delta \phi=8 \pi G \mu$, where $\mu$ is the linear mass density. Hiscock (1985) obtained the exact solution of Einstein field equation assuming energy momentum tensor for the cosmic string as $T_{i}^{j}=\operatorname{diag}(1,0,0,1) F(r)$. Rahaman and Mal (2006) studied a local cosmic string in the context of Lyra geometry in normal gauge when the displacement field is constant, and shown that only exterior solution of the local string is consistent in Lyra geometry.

In this paper, we investigate some new exact solutions for local cosmic string within the framework of Lyra geometry in normal gauge when the displacement field is a function of radial coordinate $r$ with or without a source free electromagnetic field in the context of cylindrically symmetric spacetimes. In presence of an electromagnetic field, the charge density $\sigma$, in terms variable radial coordinate $r$ has been calculated.

\section{Einstein field equations}

The stationary cylindrically symmetric line element is

$d s^{2}=d t^{2}-e^{2 \psi}\left(d r^{2}+d z^{2}\right)-L d \phi^{2}+2 M d \phi d t$,

where $\psi, L, M$ are all functions of the radial coordinate $r$ alone.

The Einstein field equations for the metric (2.1) in normal gauge for Lyra's manifold, as obtained by Sen (1957) are

$R_{i j}-\frac{1}{2} R g_{i j}+\frac{3}{2}\left(\phi_{i} \phi_{j}-\frac{1}{2} g_{i j} \phi_{\alpha} \phi^{\alpha}\right)=-\chi T_{i j}$,

where $\chi=8 \pi G$, and $\phi_{i}$ is a displacement field vector defined as $\phi_{i}=(0,0,0, \beta)$, where $\beta=\beta(r)$ and other symbols have their usual meaning as in Riemannian geometry.

The energy-momentum tensor $T_{i j}$ for the local cosmic string which is infinitely long but infinitesimal thin is given by

$T_{i}^{j}=\operatorname{diag}(0,0,1,1) f(r)$,

where $f(r)$ is the energy density of the string, we can now introduce Weyl-like canonical coordinate system in this stationary case such that (Van Stockum 1937):

$L+M^{2}=r^{2}$.

The field equations (2.2) for the metric (2.1) lead to the following system of equations:

$\frac{L^{\prime}}{2 r^{3}}-\frac{L^{\prime 2}}{8 r^{4}}-\frac{M^{\prime 2}}{2 r^{2}}-\frac{3 L^{\prime}}{8 r^{4}}+\frac{M M^{\prime}}{r^{3}}+\frac{\psi^{\prime} L^{\prime}}{2 r^{2}}+\frac{\psi^{\prime} M M^{\prime}}{r^{2}}$

$$
\begin{aligned}
& -\frac{3 L^{\prime} M M^{\prime}}{4 r^{4}}-\frac{L^{\prime} M^{\prime}}{4 r^{2}}-\frac{M^{2} M^{\prime 2}}{4 r^{4}}+\frac{3 L M^{\prime 2}}{4 r^{4}} \\
= & -\frac{3 L e^{2 \psi}}{4 r^{2}} \beta^{2},
\end{aligned}
$$

$$
\begin{aligned}
\frac{L^{\prime \prime}}{2 r^{2}}+ & \frac{M M^{\prime \prime}}{r^{2}}-\frac{L^{\prime}}{2 r^{3}}-\frac{L^{\prime 2}}{8 r^{4}}+\frac{M^{\prime 2}}{2 r^{2}}+\frac{L^{\prime}}{8 r^{4}}-\frac{M M^{\prime}}{r^{3}}-\frac{\psi^{\prime} L^{\prime}}{2 r^{2}} \\
& -\frac{\psi^{\prime} M M^{\prime}}{r^{2}}+\frac{L^{\prime} M M^{\prime}}{4 r^{4}}-\frac{L^{\prime} M^{\prime}}{4 r^{2}}+\frac{M^{2} M^{\prime 2}}{4 r^{4}}+\frac{L M^{\prime 2}}{4 r^{4}} \\
= & -\frac{3 L e^{2 \psi}}{4 r^{2}} \beta^{2},
\end{aligned}
$$

$$
\begin{gathered}
-\frac{L^{\prime \prime}}{2}+\frac{L^{\prime} M M^{\prime}}{2 r^{2}}+\frac{L^{\prime 2}}{4 r^{2}}+\psi^{\prime \prime} L+\frac{L^{\prime \prime} L}{2 r^{2}}+\frac{L M M^{\prime \prime}}{r^{2}}-\frac{L L^{\prime}}{2 r^{3}} \\
-\frac{L L^{\prime 2}}{8 r^{4}}+\frac{L L^{\prime} M M^{\prime}}{4 r^{4}}+\frac{L L^{\prime}}{8 r^{4}}-\frac{L M M^{\prime}}{r^{3}}-\frac{L L^{\prime} M^{\prime}}{4 r^{2}} \\
+\frac{L M^{2} M^{\prime 2}}{4 r^{4}}+\frac{L^{2} M^{\prime 2}}{4 r^{4}}=\chi F(r) e^{2 \psi} L-\frac{3 L^{2}}{4 r^{2}} \beta^{2}
\end{gathered}
$$

$$
\begin{aligned}
\psi^{\prime \prime}+ & \frac{L^{\prime \prime}}{2 r^{2}}+\frac{M M^{\prime \prime}}{r^{2}}-\frac{L^{\prime}}{2 r^{3}}-\frac{L^{\prime 2}}{8 r^{4}}+\frac{L^{\prime} M M^{\prime}}{4 r^{4}}+\frac{L^{\prime}}{8 r^{4}} \\
& -\frac{M M^{\prime}}{r^{3}}-\frac{L^{\prime} M^{\prime}}{4 r^{2}}+\frac{M^{2} M^{\prime 2}}{4 r^{4}}+\frac{L M^{\prime 2}}{4 r^{4}} \\
= & \chi F(r) e^{2 \psi}-\frac{3 e^{2 \psi}}{2} \beta^{2}+\frac{3 e^{2 \psi} L}{4 r^{2}} \beta^{2},
\end{aligned}
$$

$$
\begin{aligned}
M^{\prime \prime} & -\frac{L^{\prime} M^{\prime}}{2 r^{2}}-2 M \psi^{\prime \prime}-\frac{M L^{\prime \prime}}{r^{2}}-\frac{2 M^{2} M^{\prime \prime}}{r^{2}}+\frac{M L^{\prime}}{r^{3}} \\
& +\frac{M L^{\prime 2}}{4 r^{4}}-\frac{M M^{\prime 2}}{r^{2}}-\frac{L^{\prime} M^{2} M^{\prime}}{2 r^{4}}-\frac{M L^{\prime}}{4 r^{4}} \\
& +\frac{2 M^{2} M^{\prime}}{r^{3}}+\frac{L^{\prime} M M^{\prime}}{2 r^{2}}-\frac{M^{3} M^{\prime 2}}{2 r^{4}}-\frac{L M M^{\prime 2}}{2 r^{4}} \\
= & \frac{3 M L e^{2 \psi}}{2 r^{2}} \beta^{2} .
\end{aligned}
$$

Here a prime represents differentiation with respect to the radial co ordinate $r$.

\section{String solutions in the absence of an electromagnetic field}

The field equations (2.5)-(2.9) constitute a system of five equations with unknown parameters $\psi, L, M, F(r)$, and $\beta$. Therefore some additional constraint equations relating these parameters are required to obtain explicit solutions of 
the system of the field equations. Without loss of generality we may assume that

$M=a r^{2}$,

where $a$ is any arbitrary constant. Using (2.5) and (2.6), we get

$\psi^{\prime}=\frac{1}{2 r^{2}}-\frac{1}{2 r}-a^{2}+3 a^{2} r-4 a^{4} r^{3}$.

On integration, (3.2) yields

$\psi=-\frac{1}{2 r}-\ln \sqrt{r}-a^{2} r+\frac{3}{2} a^{2} r^{2}-a^{4} r^{4}+b$,

where $b$ is an arbitrary integration constant, and is taken to be zero. Thus

$\psi=-\frac{1}{2 r}-\ln \sqrt{r}-a^{2} r+\frac{3}{2} a^{2} r^{2}-a^{4} r^{4}$, and

$L=r^{2}-a^{2} r^{4}$

The cylindrically symmetric cosmological model for the local string in the absence of an electromagnetic field is given by the metric

$$
\begin{aligned}
d s^{2}= & d t^{2}-\exp \left(-\frac{1}{r}-\ln r-2 a^{2} r+3 a^{2} r^{2}-2 a^{4} r^{4}\right) \\
& \times\left(d r^{2}+d z^{2}\right) \\
& -r^{2}\left(1-a^{2} r^{2}\right) d \phi^{2}+2 a r^{2} d \phi d t
\end{aligned}
$$

The physical and kinematical parameters (Ellis 1971) for model (3.5) have the following expressions:

$\chi F(r)=p(r) \exp \left(\frac{1}{r}+\ln r+2 a^{2} r-3 a^{2} r^{2}+2 a^{4} r^{4}\right)+q(r) \exp 2\left(\frac{1}{r}+\ln r+2 a^{2} r-3 a^{2} r^{2}+2 a^{4} r^{4}\right)$

where

$p(r)=\frac{\left(64 a^{6} r^{7}-8 a^{5} r^{7}-76 a^{4} r^{5}+12 a^{3} r^{5}+2 a^{4} r^{4}+28 a^{2} r^{3}-4 a r^{3}+a^{2} r^{2}-3\right)}{4 r^{3}\left(1-a^{2} r^{2}\right)}, \quad r^{2}=1 / a^{2}$,

and

$q(r)=\frac{\left(60 a^{4} r^{5}+4 a^{2} r^{3}+2 a^{2} r^{2}-4 a^{2} r-4 r+3\right)}{4 r^{3}}, \quad \beta^{2}=\frac{4 q(r)}{3\left(1-a^{2} r^{2}\right)} \exp \left(\frac{1}{r}+\ln r+2 a^{2} r-3 a^{2} r^{2}+2 a^{4} r^{4}\right)$

It should be noted that the universe exhibits initial singularity of the BARREL-type at $r=0$. At the initial singularity $r=0$, the physical parameters $F(r)$, and $\beta$ tend to a finite limit. So the energy density of the string, and gauge function are finite at the epoch $r=0$. Moreover $F(r)$, and $\beta$ tend to infinity as $r \rightarrow \infty$. Thus the gauge function is infinitely large at the epoch $r=\infty$. Therefore the model is well behaved in the range $0<r<\infty$ for suitably chosen value of $a$.

Spatial volume:

$V^{3}=r\left(1-a^{2} r^{2}\right)^{1 / 2} \exp \left(3 a^{2} r^{2}-\frac{1}{r}-\ln r-2 a^{2} r-2 a^{4} r^{4}\right)$,

the spatial volume $V$ tends to zero as $r \rightarrow 0$, and if the radial coordinate $r$ increases then $V$ increases monotonically and tends to infinity as $r \rightarrow \infty$. The proper radial distance $R$ is given by (Som and Raychaudhury 1968)

$$
\begin{aligned}
R & =\int e^{\psi} d r \\
& =\int \exp \left(-\frac{1}{2 r}-\ln \sqrt{r}-a^{2} r+\frac{3}{2} a^{2} r^{2}-a^{4} r^{4}\right) d r
\end{aligned}
$$

the proper radial distance $R$ tends to zero as $r \rightarrow 0$, and $R \rightarrow \infty$ as $r \rightarrow \infty$.

\section{String solutions in the presence of an electromagnetic field}

The Einstein field equations in normal gauge for Lyra's manifold in the presence of an electromagnetic field are given by 
$R_{i j}-\frac{1}{2} R g_{i j}+\frac{3}{2}\left(\phi_{i} \phi_{j}-\frac{1}{2} g_{i j} \phi_{\alpha} \phi^{\alpha}\right)=-\chi\left(T_{i j}+E_{i j}\right)$,

where $T_{i j}$ is given by (2.3), and

$$
\begin{aligned}
& E_{i j}=-F_{i k} F_{j}^{k}+\frac{1}{4} g_{i j} F_{a b} F^{a b}, \\
& F_{; j}^{i j}=4 \pi J^{i}=4 \pi \sigma v^{i}
\end{aligned}
$$

and

$$
F_{[i j ; k]}=0,
$$

where $\sigma$ is the proper charge density.

Now let us introduce the following assumptions:

- The matter is at rest in the coordinate system of the metric (2.1) so that

$$
v^{i}=(0,0,0,1) \text {. }
$$

- The electromagnetic field is such that except $F^{13}$ (= $\left.-F^{31}\right)$ and $F^{14}\left(=-F^{41}\right)$, all other contravariant components of $F^{i j}$ vanish in the coordinate system of (2.1).

- The Lorentz force $F^{i j} v_{j}$ vanishes every where.

In view of first assumption, (4.3) yields

$F^{31}=\frac{K}{r} e^{-2 \psi}, \quad$ where $K$ is a constant.

Now from third assumption

$$
F^{14}=-F^{13} v_{3}=\frac{K}{r} e^{-2 \psi} M=\frac{M}{r} K e^{-2 \psi} .
$$

The non-vanishing field equations (4.1) for this case are the following:

$$
\begin{aligned}
\frac{L^{\prime}}{2 r^{3}}- & \frac{L^{\prime 2}}{8 r^{4}}-\frac{M^{\prime 2}}{2 r^{2}}-\frac{3 L^{\prime}}{8 r^{4}}+\frac{M M^{\prime}}{r^{3}}+\frac{\psi^{\prime} L^{\prime}}{2 r^{2}}+\frac{\psi^{\prime} M M^{\prime}}{r^{2}} \\
& -\frac{3 L^{\prime} M M^{\prime}}{4 r^{4}}-\frac{L^{\prime} M^{\prime}}{4 r^{2}}-\frac{M^{2} M^{\prime 2}}{4 r^{4}}+\frac{3 L M^{\prime 2}}{4 r^{4}} \\
= & -\frac{3 L e^{2 \psi}}{4 r^{2}} \beta^{2}-\frac{1}{2} \chi K^{2}, \\
\frac{L^{\prime \prime}}{2 r^{2}}+ & \frac{M M^{\prime \prime}}{r^{2}}-\frac{L^{\prime}}{2 r^{3}}-\frac{L^{\prime 2}}{8 r^{4}}+\frac{M^{\prime 2}}{2 r^{2}}+\frac{L^{\prime}}{8 r^{4}}-\frac{M M^{\prime}}{r^{3}}-\frac{\psi^{\prime} L^{\prime}}{2 r^{2}} \\
& -\frac{\psi^{\prime} M M^{\prime}}{r^{2}}+\frac{L^{\prime} M M^{\prime}}{4 r^{4}}-\frac{L^{\prime} M^{\prime}}{4 r^{2}}+\frac{M^{2} M^{\prime 2}}{4 r^{4}}+\frac{L M^{\prime 2}}{4 r^{4}} \\
= & -\frac{3 L e^{2 \psi}}{4 r^{2}} \beta^{2}+\frac{1}{2} \chi K^{2}, \\
-\frac{L^{\prime \prime}}{2} & +\frac{L^{\prime} M M^{\prime}}{2 r^{2}}+\frac{L^{\prime 2}}{4 r^{2}}+\psi^{\prime \prime} L+\frac{L^{\prime \prime} L}{2 r^{2}}+\frac{L M^{\prime \prime}}{r^{2}}-\frac{L L^{\prime}}{2 r^{3}}
\end{aligned}
$$

$$
\begin{aligned}
& -\frac{L L^{\prime 2}}{8 r^{4}}+\frac{L L^{\prime} M M^{\prime}}{4 r^{4}}+\frac{L L^{\prime}}{8 r^{4}}-\frac{L M M^{\prime}}{r^{3}} \\
& -\frac{L L^{\prime} M^{\prime}}{4 r^{2}}+\frac{L M^{2} M^{\prime 2}}{4 r^{4}}+\frac{L^{2} M^{\prime 2}}{4 r^{4}} \\
= & \chi F(r) e^{2 \psi} L-\frac{1}{2} \chi K^{2}\left(M^{2}+r^{2}\right)-\frac{3 L^{2}}{4 r^{2}} \beta^{2}, \\
\psi^{\prime \prime}+ & \frac{L^{\prime \prime}}{2 r^{2}}+\frac{M M^{\prime \prime}}{r^{2}}-\frac{L^{\prime}}{2 r^{3}}-\frac{L^{\prime 2}}{8 r^{4}}+\frac{L^{\prime} M M^{\prime}}{4 r^{4}}+\frac{L^{\prime}}{8 r^{4}} \\
& -\frac{M M^{\prime}}{r^{3}}-\frac{L^{\prime} M^{\prime}}{4 r^{2}}+\frac{M^{2} M^{\prime 2}}{4 r^{4}}+\frac{L M^{\prime 2}}{4 r^{4}} \\
= & \chi F(r) e^{2 \psi}-\frac{3 e^{2 \psi}}{2} \beta^{2}+\frac{3 e^{2 \psi} L}{4 r^{2}} \beta^{2}+\frac{1}{2} \chi K^{2} e^{-2 \psi},
\end{aligned}
$$

$$
\begin{aligned}
M^{\prime \prime} & -\frac{L^{\prime} M^{\prime}}{2 r^{2}}-2 M \psi^{\prime \prime}-\frac{M L^{\prime \prime}}{r^{2}}-\frac{2 M^{2} M^{\prime \prime}}{r^{2}}+\frac{M L^{\prime}}{r^{3}} \\
& +\frac{M L^{\prime 2}}{4 r^{4}}-\frac{M M^{\prime 2}}{r^{2}}-\frac{L^{\prime} M^{2} M^{\prime}}{2 r^{4}}-\frac{M L^{\prime}}{4 r^{4}} \\
& +\frac{2 M^{2} M^{\prime}}{r^{3}}+\frac{L^{\prime} M M^{\prime}}{2 r^{2}}-\frac{M^{3} M^{\prime 2}}{2 r^{4}}-\frac{L M M^{\prime 2}}{2 r^{4}} \\
= & \frac{3 M L e^{2 \psi}}{2 r^{2}} \beta^{2}-\chi K^{2} M .
\end{aligned}
$$

Assuming $L+M^{2}=r^{2}$ and $M=a r^{2}$, Combining (4.7)(4.8), we have

$\frac{2 \psi^{\prime}}{r}=\frac{1}{r^{3}}-\frac{1}{r^{2}}-\frac{2 a^{2}}{r}+6 a^{2}-8 a^{4} r^{2}-\chi K^{2}$

Integrating (4.12) which yields

$\psi=-\frac{1}{2 r}-\ln \sqrt{r}-a^{2} r+\frac{3}{2} a^{2} r^{2}-a^{4} r^{4}-\frac{1}{4} \chi K^{2} r^{2}$,

where arbitrary integration constant is taken to be zero.

The cylindrically symmetric cosmological model for the local string in the presence of an electromagnetic field is given by the metric

$$
\begin{aligned}
d s^{2}= & d t^{2}-\exp \left(-\frac{1}{r}-\ln r-2 a^{2} r+3 a^{2} r^{2}-2 a^{4} r^{4}\right. \\
& \left.-\frac{1}{2} \chi K^{2} r^{2}\right)\left(d r^{2}+d z^{2}\right) \\
& -r^{2}\left(1-a^{2} r^{2}\right) d \phi^{2}+2 a r^{2} d \phi d t
\end{aligned}
$$

The physical and kinematical parameters for model (4.14) have the following expressions: 


$$
\begin{aligned}
\chi F(r)= & P(r) \exp \left(\frac{1}{r}+\ln r+2 a^{2} r-3 a^{2} r^{2}+2 a^{4} r^{4}-\frac{1}{2} \chi K^{2} r^{2}\right) \\
& +Q(r) \exp 2\left(\frac{1}{r}+\ln r+2 a^{2} r-3 a^{2} r^{2}+2 a^{4} r^{4}+\frac{1}{2} \chi K^{2} r^{2}\right)
\end{aligned}
$$

where

$$
\begin{aligned}
& P(r)=\frac{\left(64 a^{6} r^{7}-8 a^{5} r^{7}-76 a^{4} r^{5}+12 a^{3} r^{5}+2 a^{4} r^{4}+28 a^{2} r^{3}-4 a r^{3}+a^{2} r^{2}-3+2 \chi K^{2} r^{3}\left(1+a^{2} r^{2}\right)\right)}{4 r^{3}\left(1-a^{2} r^{2}\right)}, \\
& r^{2}=1 / a^{2}
\end{aligned}
$$

and

$$
\begin{aligned}
& Q(r)=\frac{\left(60 a^{4} r^{5}+4 a^{2} r^{3}+2 a^{2} r^{2}-4 a^{2} r-4 r+3+2 \chi K^{2} r^{3}\right)}{4 r^{3}} \\
& \beta^{2}=\frac{4 Q(r)}{3\left(1-a^{2} r^{2}\right)} \exp \left(\frac{1}{r}+\ln r+2 a^{2} r-3 a^{2} r^{2}+2 a^{4} r^{4}+\frac{1}{2} \chi K^{2} r^{2}\right)
\end{aligned}
$$

In this case, the universe exhibits initial singularity of the BARREL-type at $r=0$. The physical parameters $F(r)$, and $\beta$ tend to a finite limit at the initial moment $r=0$. So the energy density of the string, and gauge function are finite at the initial singularity. Moreover $F(r)$, and $\beta$ tend to infinity as $r \rightarrow \infty$. Therefore the gauge function is infinitely large at the epoch $r=\infty$. For suitably chosen value of $a$, the model (4.14) is well behaved in the interval $0<r<\infty$.

$$
\begin{aligned}
V^{3}= & r\left(1-a^{2} r^{2}\right)^{1 / 2} \\
& \times \exp \left(3 a^{2} r^{2}-\frac{1}{r}-\ln r-2 a^{2} r-2 a^{4} r^{4}\right. \\
& \left.+\frac{1}{2} \chi K^{2} r^{2}\right)
\end{aligned}
$$

the spatial volume $V$ tends to zero as $r \rightarrow 0$, and if the radial coordinate $r$ increases then $V$ increases monotonically and tends to infinity as $r \rightarrow \infty$.

$$
\begin{aligned}
R=\int e^{\psi} d r= & \int \exp \left(-\frac{1}{2 r}-\ln \sqrt{r}-a^{2} r+\frac{3}{2} a^{2} r^{2}\right. \\
& \left.-a^{4} r^{4}+\frac{1}{4} \chi K^{2} r^{2}\right) d r
\end{aligned}
$$

the proper radial distance $R$ tends to zero as $r \rightarrow 0$, and $R \rightarrow \infty$ as $r \rightarrow \infty$. Also the equation

$F_{; 1}^{41}=4 \pi \sigma$

remains alone. From (4.6), the charge density $\sigma$ is given by

$$
\begin{aligned}
4 \pi \sigma= & -2 a K e^{-2 \psi} \\
= & -2 a K \exp \left(\frac{1}{r}+\ln r+2 a^{2} r-3 a^{2} r^{2}+2 a^{4} r^{4}\right. \\
& \left.+\frac{1}{2} \chi K^{2} r^{2}\right) .
\end{aligned}
$$

This model is filled with a stationary charged dust $(q=$ $\Theta=0$ ) (De and Raychaudhury 1968), where $q$ and $\Theta$ denote shear scalar and expansion scalar respectively. For $a<0$, the charge density $\sigma$, which is a function of $r$, increases exponentially as $r$ increases. The charge density is finite at the initial epoch $r=0$, and infinite at the epoch $r=\infty$.

\section{Conclusions}

In this paper, we have studied that the solutions of Einstein field equations for cylindrically symmetric metric for local string within the framework of Lyra's geometry are consistent in the presence as well as absence of an electromagnetic field. Both models have initial singularity of the BARRELtype at $r=0$. In case of both models, the energy density of the string, and gauge function are finite at the initial epoch $r=0$, and infinite at the epoch $r=\infty$. Thus gauge functions are infinitely large at the epoch $r=\infty$ for both models. The spatial volumes and proper radial distances of both models tend to zero at the singularity and tend to infinity as $r \rightarrow \infty$.

The model (4.14) is filled with a stationary charged dust. For $a<0$, the charge density $\sigma$, which is a function of $r$, increases exponentially as $r$ increases. The charge density 
is finite at the initial epoch $r=0$, and infinite at the epoch $r=\infty$. For suitably chosen value of $a$, both the models are well behaved in the interval $0<r<\infty$. Also these models have rigid rotation (i.e., vanishing shear and expansion) which gives rise to stationary solutions and cylindrically symmetric and vanishing of Lorentz force.

Acknowledgements The author expresses his thanks to Prof. Ranjit Singh, Director, and Prof. Raj Senani, Head, School of Applied Sciences, N.S.I.T., New Delhi, India for providing necessary facilities during the work.

Open Access This article is distributed under the terms of the Creative Commons Attribution Noncommercial License which permits any noncommercial use, distribution, and reproduction in any medium, provided the original author(s) and source are credited.

\section{References}

Beesam, A.: Aust. J. Phys. 11, 34 (1988)

De, U.K., Raychaudhury, A.K.: Proc. R. Soc. A 303, 97 (1968)

Ellis, G.F.R.: General Relativity and Cosmology. Academic Press, New York (1971)

Halford, W.D.: Aust. J. Phys. 23, 863 (1970)

Hiscock, W.A.: Phys. Rev. D 31, 3288 (1985)

Kibble, T.W.B.: J. Phys. A 9, 1387 (1976)

Lyra, G.: Math. Z 54, 52 (1951)

Rahaman, F., Mal, S.: Astrophys. Space Sci. 302, 3 (2006)

Sen, D.K.: Z. Phys. 149, 311 (1957)

Singh, J.K., Shri Ram: Nuovo Cim. B 112, 1157 (1997)

Singh, J.K.: Nuovo Cim. B 118, 27 (2003)

Singh, T., Singh, G.P.: Nuovo Cim. B 106, 617 (1991)

Som, M.M., Raychaudhury, A.K.: Proc. R. Soc. A 304, 81 (1968)

Van Stockum, W.J.: Proc. R. Soc. Edinb. 57, 135 (1937)

Vilenkin, A.: Phys. Rev. D 23, 852 (1981)

Zeldovich, B.: Mon. Not. R. Astron. Soc. 192, 663 (1980) 